hep-th/0109108

UA/NPPS-11-2001

\title{
Semiclassical Corrections to the Bekenstein-Hawking entropy of the BTZ Black Hole via Self-Gravitation
}

\author{
Elias C. Vagenas \\ University of Athens \\ Physics Department \\ Nuclear and Particle Physics Section \\ Panepistimioupolis, Ilisia 15771 \\ Athens, Greece
}

\begin{abstract}
Hawking radiation is viewed as a tunnelling process. In this way the effect of selfgravitation gives rise to semiclassical corrections to the entropy of the $(2+1)$ BTZ black hole. The modified entropy, due to specific modelling of the self-gravitation effect, of the $(2+1)$ BTZ black hole is evaluated. To first order in $\omega$ which is a shell of energy radiated outwards the event horizon of the BTZ black hole, modified entropy is proportional to the horizon. In this semiclassical analysis, corrections to the Bekenstein-Hawking formula $S_{B H}=\mathcal{A}_{H} / 4 l_{P}^{2}$ are found to be negative and the proportionality factor connecting the modified entropy, $S_{b h}$, of the $(2+1)$ BTZ black hole to the Bekenstein-Hawking entropy, $S_{B H}$, is evaluated to first order in $\omega$.
\end{abstract}

\footnotetext{
${ }^{1}$ hvagenas@cc.uoa.gr
} 
In this work we apply the Keski-Vakkuri, Kraus and Wilczek [1, 2, 3, [1] methodology to the $(2+1)$ BTZ black hole. The idea of KKW method is to view the BTZ black hole background as dynamical by treating the Hawking radiation [5] as a tunnelling process [6, 7, 8, 9, 10]. The energy conservation is the key to this description. The total (ADM) mass is kept fixed while the mass of the BTZ black hole decreases due to the emitted radiation. The effect of self gravitation gives rise to additional terms in the BekensteinHawking area formula concerning the BTZ black hole entropy.

The black hole solutions of Bañados, Teitelboim and Zanelli [11, 12] in $(2+1)$ spacetime dimensions are derived from a three dimensional theory of gravity:

$$
S=\int d x^{3} \sqrt{-g}\left({ }^{(3)} R+2 \Lambda\right)
$$

with a negative cosmological constant $(\Lambda>0)$.

The corresponding line element is:

$$
d s^{2}=-\left(-M+\Lambda r^{2}+\frac{J^{2}}{4 r^{2}}\right) d t^{2}+\frac{d r^{2}}{\left(-M+\Lambda r^{2}+\frac{J^{2}}{4 r^{2}}\right)}+r^{2}\left(d \theta-\frac{J}{2 r^{2}} d t\right)^{2}
$$

with $M$ the ADM mass, $J$ the angular momentum (spin) of the BTZ black hole and $-\infty<t<+\infty, 0 \leq r<+\infty$.

For the positive mass black hole spectrum with spin $(J \neq 0)$, the line element (2) has two horizons:

$$
r_{ \pm}^{2}=\frac{M \pm \sqrt{M^{2}-\Lambda J^{2}}}{2 \Lambda}
$$

with $r_{+}, r_{-}$the outer and inner horizon respectively.

The area $\mathcal{A}_{H}$ and Hawking temperature $T_{H}$ of the event (outer) horizon are [13, 14]:

$$
\begin{aligned}
\mathcal{A}_{H} & =2 \pi\left(\frac{M+\sqrt{M^{2}-\Lambda J^{2}}}{2 \Lambda}\right)^{1 / 2} \\
& =2 \pi r_{+} \\
T_{H} & =\frac{\sqrt{2 \Lambda}}{2 \pi} \frac{\sqrt{M^{2}-\Lambda J^{2}}}{\left(M+\sqrt{M^{2}-\Lambda J^{2}}\right)^{1 / 2}} \\
& =\frac{\Lambda}{2 \pi}\left(\frac{r_{+}^{2}-r_{-}^{2}}{r_{+}}\right) .
\end{aligned}
$$

The entropy of the spinning BTZ black hole is:

$$
S_{b h}=4 \pi r_{+}
$$


and if we reinstate the Planck units (since in BTZ units $8 \hbar G=1$ ) we get:

$$
S_{b h}=\frac{1}{4 \hbar G} \mathcal{A}_{H}=S_{B H}
$$

which is the well-known Bekenstein-Hawking area formula $\left(S_{B H}\right)$ for the entropy [?, 15, 5, 16] (or [17] where the formula is proven by counting excited states).

In order to apply the idea of Keski-Vakkuri, Kraus and Wilczek to the positive mass BTZ black hole spectrum with fixed $J$ we have to make a coordinate transformation. We choose the Painlevé coordinates [18] (utilized recently in 19 for black hole backgrounds) which are non-singular on the outer horizon $\left(r_{+}\right)$. We are thus able to deal with phenomena for which the main contribution comes from the outer horizon.

We introduce the time coordinate $\tau_{P}$ and the angular coordinate $\theta_{P}$ by imposing the ansätz:

$$
\begin{aligned}
& \sqrt{g(r)} d t=\sqrt{g(r)} d \tau_{P}-\frac{\sqrt{1-g(r)}}{\sqrt{g(r)}} d r \\
& \sqrt{g(r)} d \theta=\sqrt{g(r)} d \theta_{P}-\left(\frac{J}{2 r^{2}}\right) \frac{\sqrt{1-g(r)}}{\sqrt{g(r)}} d r
\end{aligned}
$$

where

$$
g(r)=-M+\Lambda r^{2}+\frac{J^{2}}{4 r^{2}}
$$

The line element (2) is now written as:

$$
\begin{aligned}
d s^{2}= & -g(r) d \tau_{P}^{2}+2 \sqrt{1-g(r)} d \tau_{P} d r+d r^{2}+\frac{J^{2}}{4 r^{2}} d \tau_{P}^{2}-J d \tau_{P} d \theta_{P}+r^{2} d \theta_{P}^{2} \\
= & -\left(-M+\Lambda r^{2}\right) d \tau_{P}^{2}+2 \sqrt{1+M-\Lambda r^{2}-\frac{J^{2}}{4 r^{2}}} d \tau_{P} d r+d r^{2} \\
& +r^{2} d \theta_{P}^{2}-J d \tau_{P} d \theta_{P} .
\end{aligned}
$$

It is obvious from the above expression that there is no singularity at the points $r_{+}$and $r_{-}$. The radial $(\theta=$ constant $)$ null $\left(d s^{2}=0\right)$ geodesics followed by a massless particle are:

$$
\dot{r} \equiv \frac{d r}{d \tau_{P}}= \pm \sqrt{1-\frac{J^{2}}{4 r^{2}}}-\sqrt{1+M-\Lambda r^{2}-\frac{J^{2}}{4 r^{2}}}
$$

where the signs + and - correspond to the outgoing and ingoing geodesics, respectively, under the assumption that $\tau_{P}$ increases towards future.

We next fix the total ADM mass and let the mass $M$ of the BTZ black hole vary. If a shell of energy (mass) $\omega$ is radiated outwards the outer horizon then the BTZ black hole mass will be reduced to $M-\omega$ and the shell of energy will travel on the modified geodesics:

$$
\dot{r}=+\sqrt{1-\frac{J^{2}}{4 r^{2}}}-\sqrt{1+(M-\omega)-\Lambda r^{2}-\frac{J^{2}}{4 r^{2}}}
$$


produced by the modified line element:

$$
\begin{aligned}
d s^{2}= & -\left(-(M-\omega)+\Lambda r^{2}\right) d \tau_{P}^{2}+2 \sqrt{1+(M-\omega)-\Lambda r^{2}-\frac{J^{2}}{4 r^{2}}} d \tau_{P} d r+d r^{2} \\
& +r^{2} d \theta_{P}^{2}-J d \tau_{P} d \theta_{P} .
\end{aligned}
$$

It is known that the emission rate from a radiating source can be expressed in terms of the imaginary part of the action for an outgoing positive-energy particle as:

$$
\Gamma=e^{-2 \operatorname{Im} S}
$$

but also in terms of the temperature and the entropy of the radiating source which in our case will be a BTZ black hole:

$$
\Gamma=e^{-\beta \omega}=e^{+\Delta S_{b h}}
$$

where $\beta$ is the inverse temperature of the BTZ black hole and $\Delta S_{b h}$ is the change in the entropy of the BTZ black hole before and after the emission of the shell of energy $\omega$ (outgoing massless particle). It is clear that if we evaluate the action then we will know the temperature and/or the change in the entropy of the BTZ black hole.

We therefore evaluate the imaginary part of the action for an outgoing positive-energy particle which crosses the event horizon outwards from:

$$
r_{i n}^{2}=r_{+}^{2}(M, \Lambda, J)=\frac{M+\sqrt{M^{2}-\Lambda J^{2}}}{2 \Lambda}
$$

to

$$
r_{\text {out }}^{2}=r_{+}^{2}(M-\omega, \Lambda, J)=\frac{(M-\omega)+\sqrt{(M-\omega)^{2}-\Lambda J^{2}}}{2 \Lambda} .
$$

The imaginary part of the action is:

$$
\operatorname{Im} S=\operatorname{Im} \int_{r_{\text {in }}}^{r_{\text {out }}} p_{r} d r=\operatorname{Im} \int_{r_{\text {in }}}^{r_{\text {out }}} \int_{0}^{p_{r}} d p_{r}^{\prime} d r .
$$

We make the transition from the momentum variable to the energy variable using Hamilton's equation $\dot{r}=\frac{d H}{d p_{r}}$ and equation (14). The result is:

$$
\operatorname{Im} S=\operatorname{Im} \int_{r_{\text {in }}}^{r_{\text {out }}} \int_{0}^{\omega} \frac{\left(-d \omega^{\prime}\right) d r}{\sqrt{1-\frac{J^{2}}{4 r^{2}}}-\sqrt{1+\left(M-\omega^{\prime}\right)-\Lambda r^{2}-\frac{J^{2}}{4 r^{2}}}}
$$

where the minus sign is due to the Hamiltonian being equal to the modified mass $H=$ $M-\omega$. This is not disturbing since $r_{\text {in }}>r_{\text {out }}$. After some calculations (involving contour integration into the lower half of $\omega^{\prime}$ plane) we get:

$$
\operatorname{Im} S=2 \pi\left\{\left[r_{\text {in }} \sqrt{1-\frac{J^{2}}{4 r_{\text {in }}^{2}}}+\frac{J}{2} \arcsin \left(\frac{J}{2 r_{\text {in }}}\right)\right]-\left[r_{\text {out }} \sqrt{1-\frac{J^{2}}{4 r_{\text {out }}^{2}}}+\frac{J}{2} \arcsin \left(\frac{J}{2 r_{\text {out }}}\right)\right]\right\} .
$$


Apparently the emission rate depends not only on the mass $M$ and angular momentum (spin) $J$ of the BTZ black hole but also on the energy $\omega$ of the emitted massless particle:

$$
\begin{aligned}
& \Gamma(\omega, M, \Lambda, J)=e^{-2 \text { ImS }} \\
= & \exp \left[4 \pi\left\{\left[r_{\text {out }} \sqrt{1-\frac{J^{2}}{4 r_{\text {out }}^{2}}}+\frac{J}{2} \arcsin \left(\frac{J}{2 r_{\text {out }}}\right)\right]-\left[r_{\text {in }} \sqrt{1-\frac{J^{2}}{4 r_{\text {in }}^{2}}}+\frac{J}{2} \arcsin \left(\frac{J}{2 r_{\text {in }}}\right)\right]\right\}\right] .
\end{aligned}
$$

Comparing (23) and (17) in which:

$$
\Delta S_{b h}=S_{b h}(\omega, M, \Lambda, J)-S_{b h}(M, \Lambda, J)
$$

we deduce that the modified entropy due to the specific modelling of the self-gravitation effect of the BTZ black hole is:

$$
S_{b h}(\omega, M, \Lambda, J)=4 \pi r_{\text {out }} \sqrt{1-\frac{J^{2}}{4 r_{\text {out }}^{2}}}+2 \pi J \arcsin \left(\frac{J}{2 r_{\text {out }}}\right)+S_{0}
$$

where $S_{0}$ is an arbitrary constant which will be evaluated.

The second term in equation (24) should be the Bekenstein-Hawking area formula:

$$
S_{b h}(M, \Lambda, J)=S_{B H}
$$

and comparing with equation (23) we obtain:

$$
S_{B H}=4 \pi r_{i n} \sqrt{1-\frac{J^{2}}{4 r_{i n}^{2}}}+2 \pi J \arcsin \left(\frac{J}{2 r_{i n}}\right)+S_{0} .
$$

Hence, the "arbitrary" constant $S_{0}$ is given as:

$$
S_{0}=S_{B H}\left[1-\sqrt{1-\frac{J^{2}}{4 r_{i n}^{2}}}-\frac{J}{2 r_{i n}} \arcsin \left(\frac{J}{2 r_{i n}}\right)\right] .
$$

Therefore, substituting expression (28) for the "arbitrary" constant $S_{0}$ in equation (25) we get the full expression for the modified entropy due to the specific modelling of the self-gravitation effect of the $(2+1)$ BTZ black hole:

$$
\begin{array}{r}
S_{b h}(\omega, M, \Lambda, J)=S_{B H}+4 \pi\left[r_{\text {out }} \sqrt{1-\frac{J^{2}}{4 r_{\text {out }}^{2}}}-r_{\text {in }} \sqrt{1-\frac{J^{2}}{4 r_{\text {in }}^{2}}}\right] \\
+2 \pi J\left[\arcsin \left(\frac{J}{2 r_{\text {out }}}\right)-\arcsin \left(\frac{J}{2 r_{\text {in }}}\right)\right] .
\end{array}
$$

We see that there are deviations from the standard result previously mentioned (see equation (6)) for a fixed BTZ black hole background. The entropy of the BTZ black hole 
is no longer the Bekestein-Hawking area formula $S_{B H}$. The corrections to the BekesteinHawking area formula for the entropy are not obviously negative. In order to examine if the modified entropy is smaller or larger compared to the entropy $S_{B H}$ for the fixed BTZ black hole background we work to first order in $\omega$.

We Taylor expand $r_{\text {out }}$ and keep up to linear terms in $\omega$. The result is:

$$
r_{\text {out }}=r_{\text {in }}(1-\epsilon \omega)
$$

where $\epsilon$ is a parameter:

$$
\epsilon=\frac{1}{2 \sqrt{M^{2}-\Lambda J^{2}}} .
$$

This parameter is small for sufficiently large mass of the BTZ black hole (as it is expected for such a semiclassical analysis here employed since the radiating matter is viewed as point particles).

Therefore, the modified entropy in (29) evaluated to first order in $\omega$ is given as:

$$
S_{b h}(\omega, M, \Lambda, J)=S_{B H}\left\{1-\epsilon \omega\left[1-\frac{J^{2}}{8 r_{i n}^{2}}\right]\right\}
$$

where

$$
\frac{J^{2}}{8 r_{i n}^{2}} \ll 1
$$

for sufficiently large mass of the BTZ black hole.

It is now obvious that to first order in $\omega$, the semiclassical corrections to the BekensteinHawking area formula for the entropy are negative. Therefore the maximum entropy that a black hole can have is the Bekenstein-Hawking entropy, $S_{B H}$, and when semiclassical effects are included the entropy decreases:

$$
S_{b h}(\omega, M, \Lambda, J) \leq S_{B H}
$$

A welcomed but not unexpected result is that equality in the above equation holds for zeroth order in $\omega$.

We also see that the modified entropy (32) of the $(2+1)$ BTZ black hole is proportional to its horizon area. The modified entropy (32) will then be written as:

$$
\begin{aligned}
S_{b h}(\omega, M, \Lambda, J) & =\alpha S_{B H} \\
& =\frac{\alpha}{4} \mathcal{A}_{H}
\end{aligned}
$$

where the dimensionless proportionality factor $\alpha$ :

$$
\alpha=\left\{1-\epsilon \omega\left[1-\frac{J^{2}}{8 r_{i n}^{2}}\right]\right\}
$$


is a function of the emitted shell's energy $\omega$ and the $(2+1)$ BTZ black hole parameters: (i) mass, (ii) angular momentum. Therefore, the modified entropy can be written as:

$$
S_{b h}(\omega, M, \Lambda, J)=\frac{1}{4} \mathcal{A}_{b h}(\omega, M, \Lambda, J)
$$

where the area of the $(2+1)$ BTZ black hole has now been modified to:

$$
\mathcal{A}_{b h}(\omega, M, \Lambda, J)=\alpha \mathcal{A}_{B H} .
$$

This is mainly the reason for the modifications to the Bekestein-Hawking entropy of the $(2+1)$ BTZ black hole due to the specific modelling of the self-gravitation effect adopted here.

\section{Acknowledgements}

The author would like to thank Ass. Professor T. Christodoulakis for useful discussions and enlightening comments. This work is financially supported in part by the University of Athens' Special Account for the Research.

\section{References}

[1] E. Keski-Vakkuri and P. Kraus, Phys. Rev. D54 (1996) 7407.

[2] P. Kraus and F. Wilczek, Nucl. Phys. B433 (1995) 403.

[3] P. Kraus and F. Wilczek, Nucl. Phys. B437 (1995) 231.

[4] E. Keski-Vakkuri and P. Kraus, Nucl. Phys. B491 (1997) 249.

[5] S.W. Hawking, Commun. Math. Phys. 43 (1975) 199.

[6] M.K. Parikh and F. Wilczek, Phys. Rev. Lett. 85 (2000) 5042.

[7] Y. Kwon, Il Nuovo Cimento B115 (2000) 469.

[8] S. Hemming and E. Keski-Vakkuri, Phys. Rev. D64 (2001) 044006.

[9] E.C. Vagenas, Phys. Lett. B503 (2001) 399.

[10] A.J.M. Medved, Class. Quant. Grav. 19 (2002) 589.

[11] M. Bañados, C. Teitelboim and J. Zanelli, Phys. Rev. Lett. 69 (1992) 1849. 
[12] M. Bañados, M. Henneaux, C. Teitelboim and J. Zanelli, Phys. Rev. D48 (1993) 1506.

[13] S. Carlip and C. Teitelboim, Phys. Rev. D51 (1995) 622.

[14] S. Carlip, Class. Quant. Grav. 12 (1995) 2853.

[15] J.D. Bekenstein, Phys. Rev. D9 (1974) 3292.

[16] S.W. Hawking, Phys. Rev. D13 (1976) 191.

[17] A. Strominger, JHEP 9802 (1998) 009.

[18] P. Painlevé, C.R. Acad. Sci.(Paris) 173 (1921) 677.

[19] P. Kraus and F. Wilczek, Mod. Phys. Lett. A9 (1994) 3713. 